Development of the Vacuum Power Flow Channel for the Mini-G

J. B. Javedani, D. A. Goerz, D. B. Reisman, T. L. Houck, M. P. Perkins, R. A. Richardson, G. E. Vogtlin

December 11, 2012

2012 International Conference on Megagauss Magnetic Field Generation and Related Topics Maui, HI, United States October 14, 2012 through October 19, 2012 
This document was prepared as an account of work sponsored by an agency of the United States government. Neither the United States government nor Lawrence Livermore National Security, LLC, nor any of their employees makes any warranty, expressed or implied, or assumes any legal liability or responsibility for the accuracy, completeness, or usefulness of any information, apparatus, product, or process disclosed, or represents that its use would not infringe privately owned rights. Reference herein to any specific commercial product, process, or service by trade name, trademark, manufacturer, or otherwise does not necessarily constitute or imply its endorsement, recommendation, or favoring by the United States government or Lawrence Livermore National Security, LLC. The views and opinions of authors expressed herein do not necessarily state or reflect those of the United States government or Lawrence Livermore National Security, LLC, and shall not be used for advertising or product endorsement purposes. 


\title{
Development of the Vacuum Power Flow Channel for the Mini-G
}

\author{
Javedani J.B., Goerz D.A., Reisman D.B., Houck T.L., Perkins M.P., Richardson R.A., Vogtlin G.E. \\ Lawrence Livermore National Laboratory \\ Livermore CA 94550 USA \\ javedani1@1lnl.gov
}

\begin{abstract}
The Mini-G explosive pulsed power system is a two-stage helical-coaxial FCG that is geometrically a half-scale version of LLNL's FFT device. The generator is capable of delivering $60 \mathrm{MA}$ currents and $10 \mathrm{MJ}$ of energy to suitable inductive loads. The Mini-G is presently used in high-energy-density physics experiments that require efficient current delivery through a vacuum power flow region to the load. As with the FFT device, the Mini-G system requires a compact, high-voltage gas-to-vacuum insulator and low-inductance vacuum power flow channel to achieve high performance and maximum energy delivery. In designing the Mini-G system, we followed the successful approach used in developing the FFT device. This included shaping the electrodes and insulators to manage electric field enhancements, applying coatings to cathode surfaces to suppress electron field emission, introducing baffles to the power flow channel to block UV, and applying coatings to electrode surfaces to absorb UV. This paper describes the design of the Mini-G vacuum interface and power flow region, and results of modeling and simulations that were done to evaluate and optimize performance. Appropriate codes were used to examine electric field enhancements, magnetic insulation, flashover inhibition and UV ray tracing in the channel. In this paper, we also present results of laboratory testing on HV vacuum insulator materials and shapes, UV induced insulator flashover, along with measurements of HV thresholds for electron emission. We also report on UV reflectance data for some of the coatings considered. To date, there have been eight experiments performed using the Mini-G system. For the first two tests, the power flow channel had an extremely low vacuum inductance of 0.9 nH. On the second Mini-G test it appeared that a partial shorting occurred in the power flow channel, limiting full energy delivery to the load. The design was modified to reduce electrical stress, improve UV attenuation, and incorporate additional diagnostics. This increased the inductance of the power flow channel to $1.5 \mathrm{nH}$. On the third Mini-G test the partial shorting reoccurred and the new diagnostics (inner Bdot probe) helped to identify the location at the vacuum insulator surface - about $10 \%$ of total current of $41 \mathrm{MA}$ was diverted into the short. Further design modifications were incorporated to decrease electrical stress across the insulator and reduce UV illumination of the insulator surface. This increased the inductance of the power flow channel to $1.9 \mathrm{nH}$. On subsequent Mini-G experiments full current delivery to the load has been achieved with no occurrence of shorting.
\end{abstract}

Keywords-Explosively Driven Magnetic Flux Compression;FCG; CMG; Power Flow;Vacuum; Insulator; low inductance load; Computaional Codes; Test Stands;

\section{INTRODUCTION}

The Mini-G explosively driven two-stage flux compression generator system, shown in Figure 1, is a nearly onehalf scale version of LLNL's FFT system [1]. The first stage helical generator is about $100 \mathrm{~cm}$ in length and $26 \mathrm{~cm}$ in diameter with an initial inductance of about $10 \mathrm{uH}$. The armature is an annealed aluminum tube, $15 \mathrm{~cm}$ in diameter and $0.953 \mathrm{~cm}$ thick, filled with $16 \mathrm{~kg}$ of PBXN110, and initiated at one end by a single detonator and plane wave HE lens. The stator is comprised of four sections starting with two wires and bifurcating to 4, 8 , and then 16 windings over the first $35 \mathrm{~cm}$ of its length. At the output end of the stator the windings attach to a conical section that couples to the input of the second stage coaxial generator. An insulator, shaped like a top hat, separates the inner and outer conductors of the two generators. This insulator is designed to hold off voltage of about $20 \mathrm{kV}$. The coaxial generator is about $100 \mathrm{~cm}$ in length and $33 \mathrm{~cm}$ in diameter, with an initial inductance of about $70 \mathrm{nH}$. This type of coaxial generator has the armature on the outside and the stator in the inside. The $25-\mathrm{cm}$ diameter armature is surrounded by $31 \mathrm{~kg}$ of pressed and machined LX-14 explosive. On the outside of the LX-14 is a steel tamper with a ring of 51 detonators installed that simultaneously initiate the explosive. As the HE burns and expands the armature is squeezed inward at the waist, collapsing on the contoured stator in a controlled zippering action to compress the magnetic flux from the input end toward the vacuum power flow channel (VPFC). Both the helical generator and the coaxial generator are filled with one atmosphere of $\mathrm{SF}_{6}$ to better withstand the electrical breakdowns due to internal electric fields at the armature/stator contact point region, as well as at the input and output insulator triple point regions. 

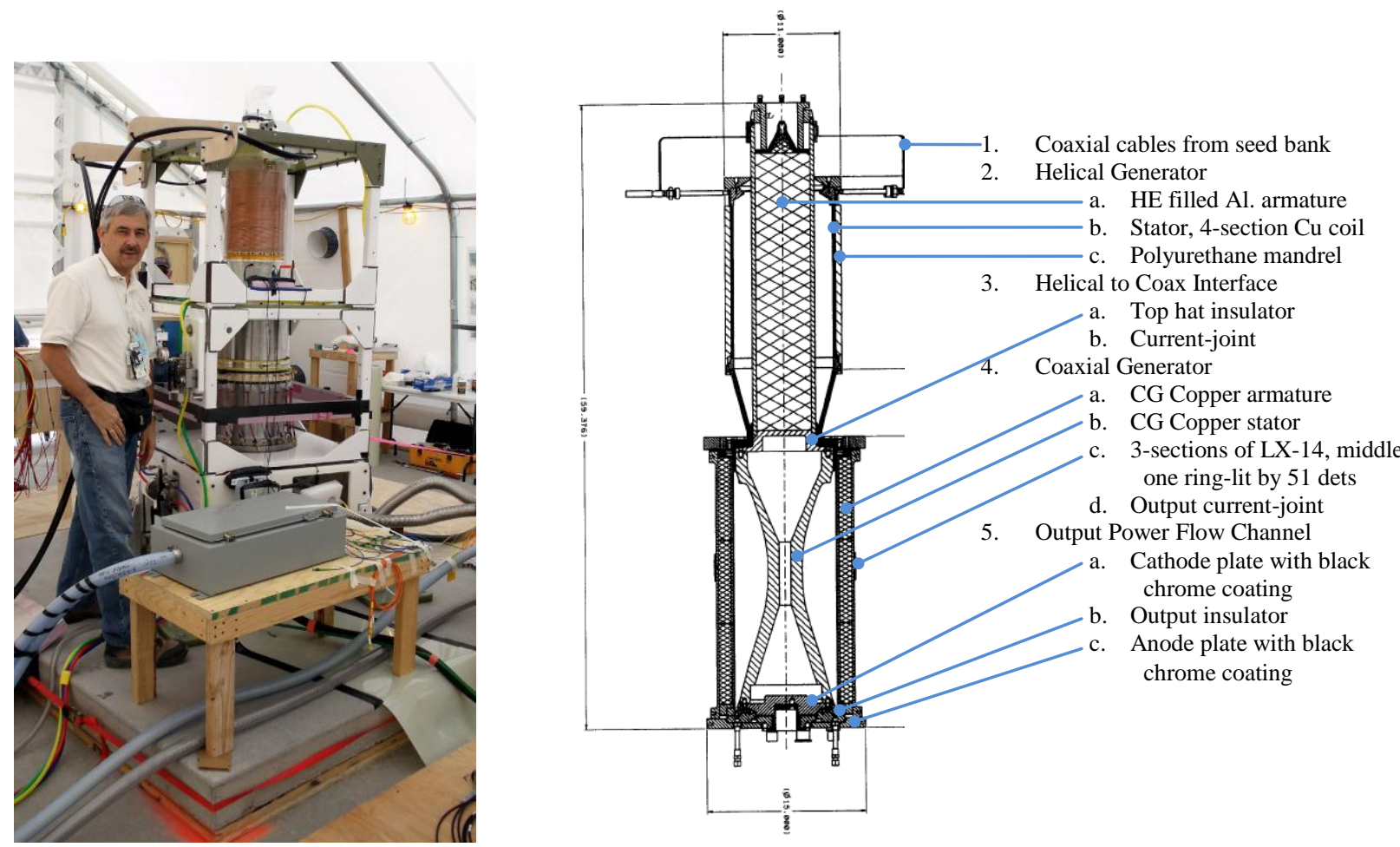

Figure 1. (left) Photo of a Mini-G prior to shot with one of the authors, Dave Goerz. (right) Cross-section drawing of Mini-G with major components identified.

In a typical shot, the helical generator is initially seeded with a current of about $70 \mathrm{kA}$ from a capacitor bank having a rise-time of about $85 \mu \mathrm{s}$. The armature detonation is set off at around $50 \mu \mathrm{s}$ to allow time for the armature to expand and crowbar the helical generator input at near peak current. The helical generator amplifies the current to about $8 \mathrm{MA}$ after $62 \mu \mathrm{s}$ from its time of crowbar. The coaxial generator in turn amplifies the current to $60 \mathrm{MA}$ after $12 \mu \mathrm{s}$ from its time of crowbar. The initial seed flux is 0.65 Weber. Due to resistive and diffusive losses in the helical generator, the flux is reduced to about 0.40 Weber in the coaxial generator when it crowbars. After the coaxial generator has functioned there is about 0.12 Weber of flux within the VPFC.

The transfer of current into a useful load region is of critical importance to any pulsed power application. The Mini-G system requires a compact, high-voltage gas-to-vacuum insulator and low-inductance VPFC to achieve high performance and maximum energy delivery. The design of the VPFC and the performance of the overall system are very much dependent on the specifics of the load requirements. Figure 2 shows the range of performance expected from the coaxial generator of the size indicated based on MHD simulation. For a seed current of $8 \mathrm{MA}$, the output current can range from 50 to $75 \mathrm{MA}$, depending on the load inductance ranging from 1 to $4 \mathrm{nH}$.
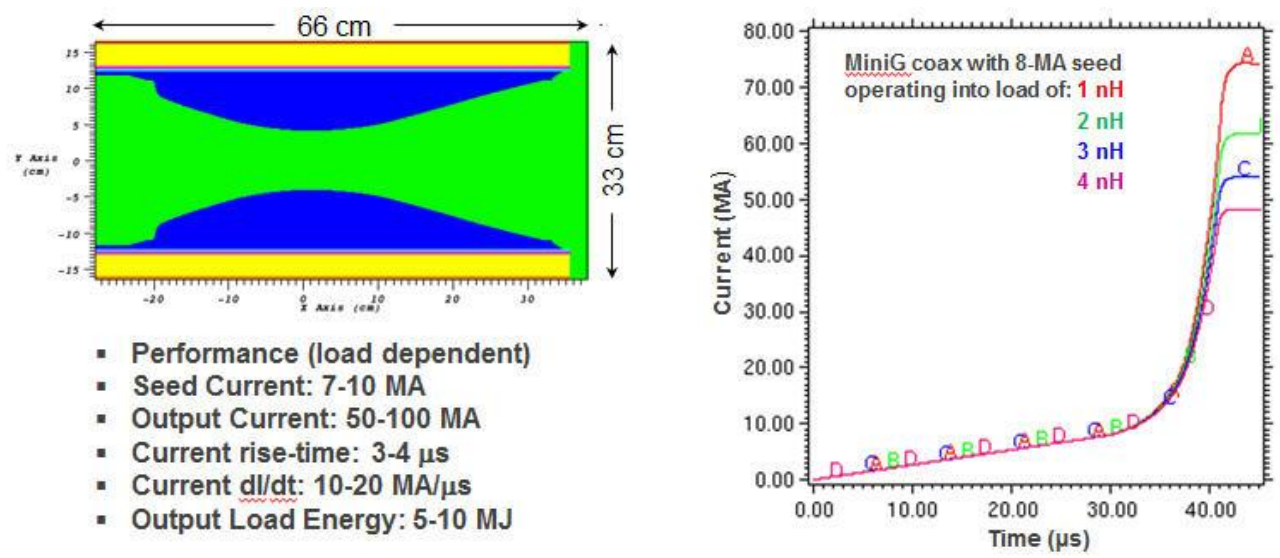

Figure 2. (left) drawing of Mini-G coaxial generator and list of performance ratings depending on load conditions. (right) MHD simulation results showing Mini-G output current for various (fixed) load inductances. 


\section{Mini-G VAcuUm Power Flow ChanNel}

In designing the Mini-G VPFC, we followed the successful approach used in developing the FFT device [2]. This included shaping the electrodes and insulators to manage electric field enhancements, applying coatings to cathode surfaces to suppress electron field emission, introducing baffles into the power flow channel to block UV, and applying coatings to electrode surfaces to absorb UV. Figure 3 shows the initial design of the Mini-G VPFC and the evolution of the design over the first set of four experiments.

For the first two Mini-G tests, the VPFC had an extremely low inductance of $0.9 \mathrm{nH}$ and incorporated a concave shaped Rexolite insulator. On the second Mini-G test it appeared that a partial shorting occurred somewhere in the power flow channel, limiting full energy delivery to the load. After that test we modified the design to reduce electrical stress, improve UV attenuation, and incorporate additional diagnostics. The changes in shape increased the inductance of the VPFC to $1.5 \mathrm{nH}$. On the third Mini-G test the partial shorting reoccurred and the new diagnostics (inner B-dot probes) helped to identify the location of the partial short to be at the vacuum insulator surface. By comparing inner and outer B-dot measurements we were able to determine that about $10 \%$ of the total 41 MA current was diverted by the breakdown. We assume that insulator surface flashover occurred due to high UV exposure. Further design modifications were incorporated to decrease electrical stress across the insulator and reduce UV illumination of the insulator surface. These changes increased the inductance of the power flow channel to $1.9 \mathrm{nH}$. On the fourth Mini-G test we achieved full current delivery to the load. On this test we estimate that the UV dose illuminating the insulator surface was less than $10 \mathrm{~mJ} / \mathrm{cm}^{2}$. We have continued to use this design on all subsequent experiments and have achieved full current delivery. We have not had the opportunity to test whether the concave insulator exposed to the same fields and UV dose would also perform as well.

\section{MODELIING AND SiMULATION OF VPFC}

During the design and development phase we applied a variety of computational tools to evaluate requirements and understand the mechanisms that could limit performance. Some of the codes we used were developed at LLNL and some are available commercially. In this section we briefly summarize the process we went through in utilizing each code, without getting into the details of the physics of the codes.

2D Arbitrary Lagrange-Eulerian (ALE) magneto-hydrodynamics (MHD) codes such as CALE [3] and ARES [4] were used to calculate the coaxial generator's time varying inductance, current, and output voltage (across the output insulator). These codes were also used in predicting the UV radiation dose due to black body emission from the heated channel walls. Figure 4 shows the ARES simulation of the armature zippering action at a few snap shots in time. This simulation shows the voltage across the insulator can reach $40 \mathrm{kV}$ at peak current (40 MA). The MHD portion of the code is used to predict the voltage but at this time is not able to handle polarization of electric field inside materials with dielectric constant other than unity.

MiniG 1-2

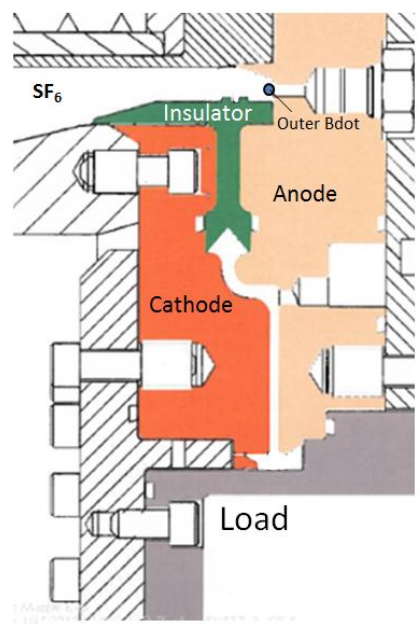

MiniG 3

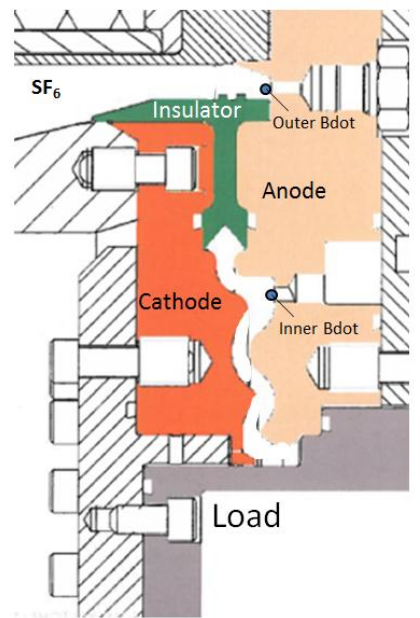

MiniG 4-xx

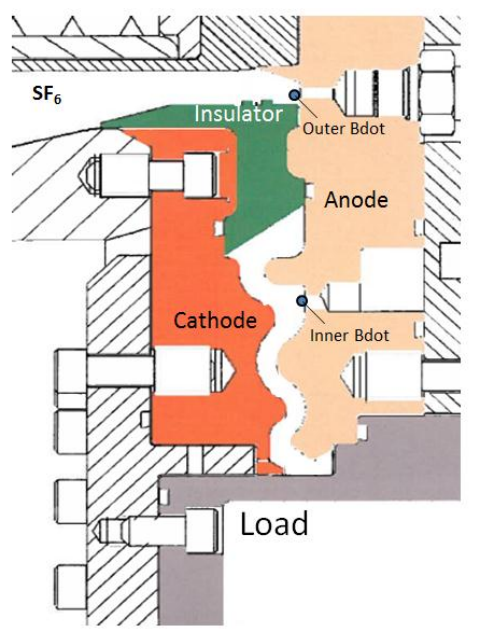

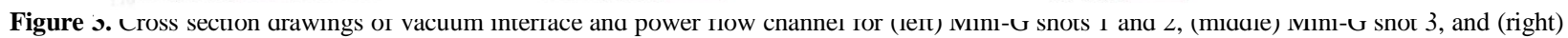
Mini-G shot 4 and beyond. 

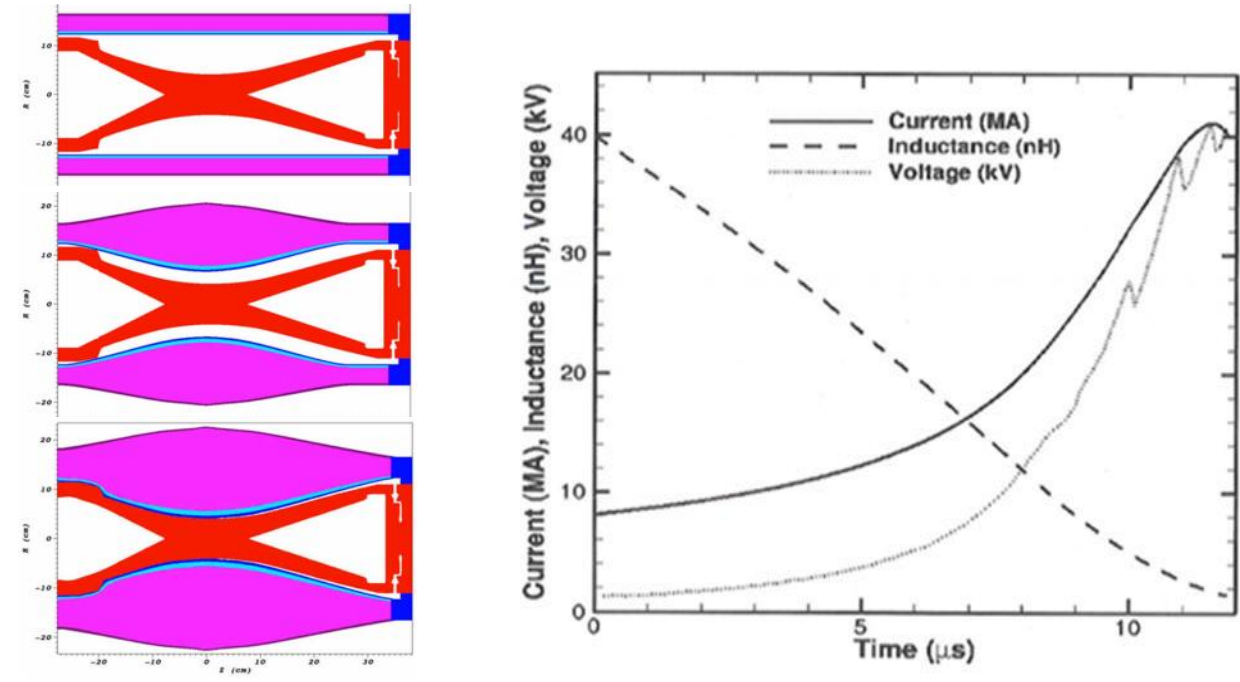

Figure 4. ARES computational modeling results of the collapsing armature (blue) and prediction of inductance, current and voltage (across the insulator) as a function of time.

The output of the ALE codes can be used with other codes such as FlexPDE [5], or Maxwell Electrostatic Solver [6] to investigate the electric field behavior and intensity with emphasis in the triple point regions of the insulator (Rexolite, $\varepsilon=2.3$ ). In FlexPDE (a partial differential solver, finite element code), we applied the Electric Vector Potential scheme, as first demonstrated by Kiuttu, to solve for the inductive electric field [7]. Kiuttu's formulation is a powerful tool for the conceptual design of coaxial generators. For a set of desired performance parameters, e.g. current, rate of change of current, and wall velocities, the electric field stresses can be determined at specific times. The wall velocities are treated as boundary conditions related to the flow of energy into or out of the simulation. Figure 5 shows FlexPDE predictions of the electric field at different times along the cathode surface in the channel of Mini-G 1, where a concave shaped insulator was used. The results of the Maxwell modeling of the same geometry is in agreement with FlexPDE lineout results of electric field in the insulator region - a region electrically far away from the centerline. Relatively high electric fields were calculated for conducting surfaces and significant fields were found for the insulator surfaces, although the triple junctions at the vacuum insulator were protected by the design geometry.

An interesting situation applies for the insulator. The electric field is not sufficient to directly pull electrons from the insulator, but UV illumination will produce free electrons at the surface that can then be accelerated by the field. For our situation there is a strong magnetic field present and any emitted electrons from the cathode will be directed away from the surface of the insulator due to E x B drift, thereby inhibiting the formation of an electron avalanche as an onset for a breakdown. Vandevender et al. in their observation of magnetic flashover inhibition of angled insulator formulated empirical criteria between applied electric field and the background magnetic field [8]:
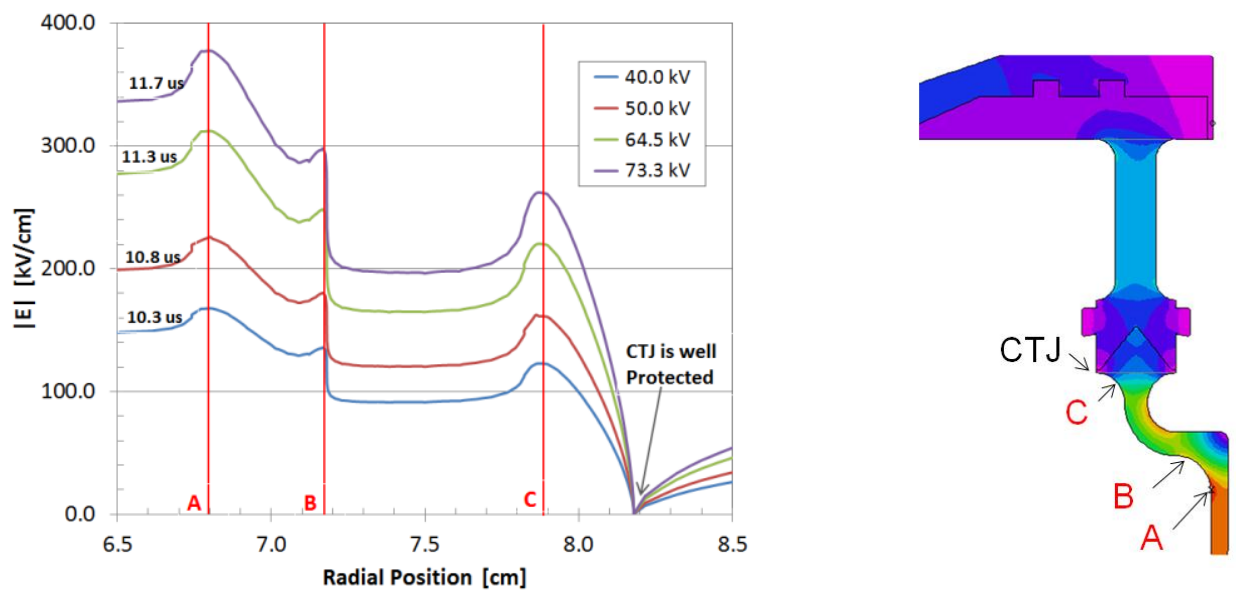

Figure 5. Shows FlexPDE modeling results of electric field along the cathode surface of VPFC at several times. 


$$
\mathrm{B} / \mathrm{E}>6.0 \times 10^{8} \text { (MKS units) }
$$

Based on this criterion, assuming an applied electric field of $400 \mathrm{kV} / \mathrm{cm}$ and a radius of $8 \mathrm{~cm}$, the electrons should experience magnetic flashover inhibition at less than 1.0 MA of generator current. However, at the time of maximum field stresses the generator current is approximately $40 \mathrm{MA}$, well above the threshold for flashover inhibition.

The effects of Magnetic Flashover Inhibition (MFI) as well as Magnetic Insulation (MI) for the VPFC were further investigated with the commercial code from Field Precision in 2D and 3D [9]. Three distinct electron emission scenarios were examined with the code: 1) emissions of electrons due to UV irradiation of the insulator surface, 2) Child-Langmuir limited electron emission from the cathode surface and 3) Fowler-Nordheim limited electron emission from the cathode surface.

In the first scenario, assuming a conversion efficiency of photons ( $>5 \mathrm{eV}$ ) to electrons of 10,000:1 [10] and the expected UV fluence (see discussion on FRED modeling below), it was calculated that at $100 \mathrm{kV}$ potential across the insulator and only 2 MA of current was required to sweep back any emitted electrons back into the insulator. See Figure 6 (left). This is consistent with the empirically driven MFI criteria observed by Vandenvender, as expressed in equation (1).

For the case of Fowler-Nordheim emission, assuming a work function of $1.4 \mathrm{eV}$ for electrons and a surface roughness of 7 , the emitted electron current is less than $30 \mathrm{~mA}$ for a $100 \mathrm{kV}$ across the channel. For the emission simulations a constant $100 \mathrm{kV}$ potential was assumed between the cathode and anode surfaces as a conservative simplification of the more complex inductive electric field. The cycloid orbital radius is about one micron, when operating at the peak generator current ensuring magnetic insulation even in the narrow portions of the channel. Current contribution from Fowler-Nordheim can be safely ignored. Ion emission from the anode surface is negligible as well, since the larger ion cycloid orbital radius is a small fraction of a millimeter.

In studying the Child-Langmuir or space charge limited emission scenario, the entire surface of the cathode was allowed to emit electrons. See Figure 6 (right). A constant $100 \mathrm{kV}$ potential was assumed between the cathode and anode surfaces and the generator current was increased until at about 1.0 MA the Larmor radius $(\sim 1 \mathrm{~mm})$ was too small to adequately resolve in the simulation grid. The large emission area and high fields $(\sim 500 \mathrm{kV} / \mathrm{cm}$ in the narrow channel) led to large currents at low generator currents/magnetic fields. As the generator current was increased, the emitted electrons were confined to orbits near the cathode surface reducing the electric field at the surface and total emitted current to $\sim 65 \mathrm{kA}$. The generator current is calculated to be approximately $40 \mathrm{MA}$ at the maximum field stresses in the VPFC. At this level of generator current, the emitted cathode current should be significantly less, cycloid orbital radius is about a micron and magnetic insulation is complete.

An effect not simulated, is the expansion of neutral plasma into the VPFC. A plausible scenario for the creation and density of the plasma was not established. However, neutral plasma is a concern as magnetic insulation would not be effective. The plasma would act as a short once the channel is crossed. The inductance and resistance associated with plasma short would have to be estimated to determine the effect on the power flow. The extremely low inductance of the VPFC provides some robustness with respect to a narrow shorting channel within the plasma. - This is a plausible hypothesis that cannot be discounted as a possibility in the partial shorting in the earliest Mini-G shots.
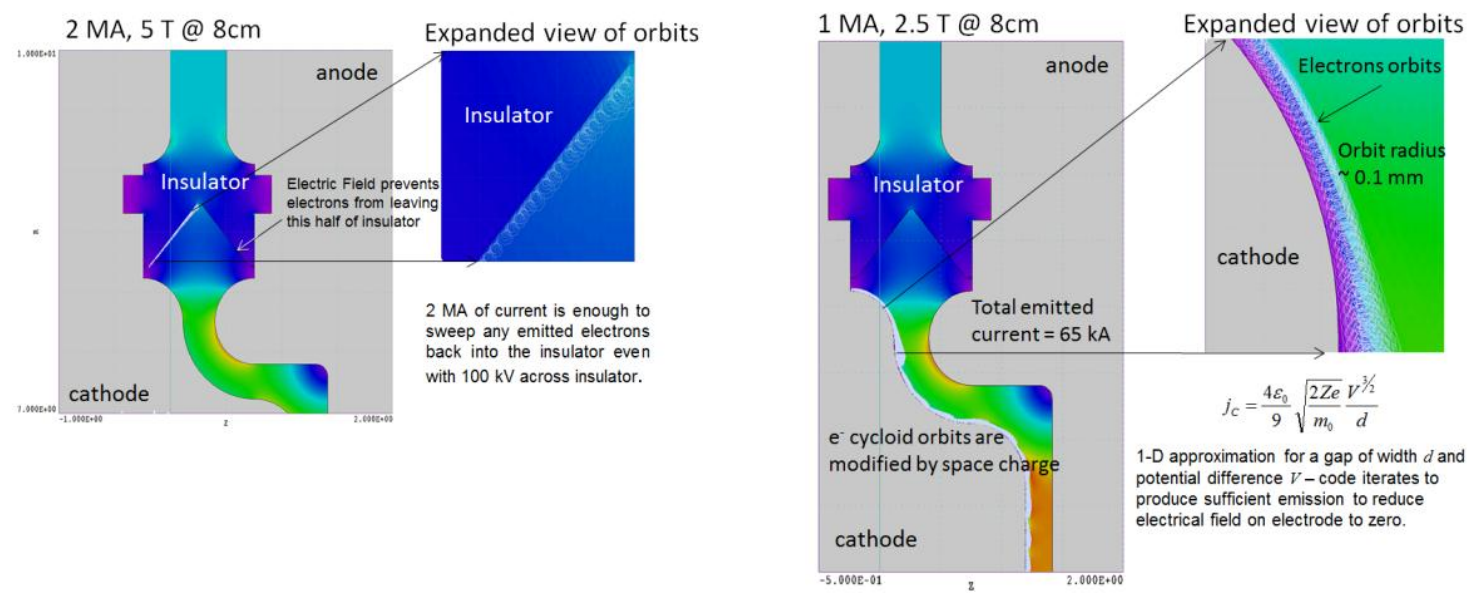

Figure 6. (left) Shows the presence of UV-induced electrons on the surface of insulator is swept back into the insulator even with $100 \mathrm{kV}$ across the insulator. (right) Shows Child-Langmuir electrons current contribution is about $6.5 \%$ of total current, but have a tight orbit of $0.1 \mathrm{~mm}-$ much smaller than the $\sim 2 \mathrm{~mm}$ gap. 

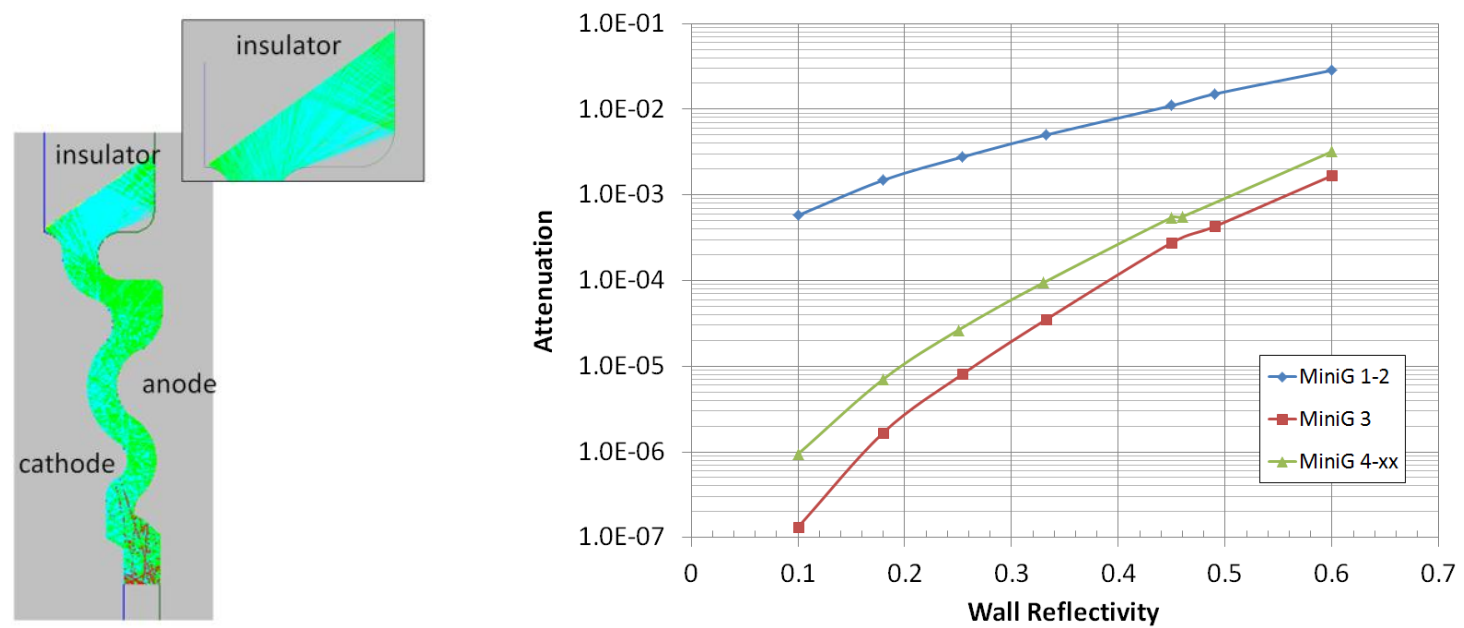

Figure 7. (left) Tracing rays with FRED for the case of Mini-G 4 channel, (right) attenuation of incident light at the insulator surface as a function of wall reflectivity for Mini-G channels.

To quantify the efficiency of the channel in attenuating photons, the VPFC was modeled with FRED ${ }^{\mathrm{TM}}$ [11], a 3D ray-tracing code, to predict the UV fluence reaching the insulator. To keep the analysis simple, we only modeled the geometry in the initial condition before distortions due to electromagnetic forces. After defining an emission region (black body source) at the bottom of the channel, we ran the code for different wall reflectance values and calculated the percent attenuation at the insulator for different configurations. The results of these runs are shown in Figure 7 (left). Of the three considered configurations, Mini-G 3 channel had the best geometry in attenuating photons as shown in Figure 7 (right). During operations the channel geometry/parameters change as the walls heat to melting temperatures and move due to electromagnetic forces. The UV source fluence increases as the channel surface heats. The effective wall reflectivity for Mini-G 1-3 is estimated to be $46 \%$ based on partial shorting of the Bdot probe diagnostics.

\section{LABORATORY TESTING}

Simulation plays a big role in the design of the channel but it is not all that goes into the design of a VPFC. Additionally, certain parameters such as insulator voltage hold off (under static conditions), electron emission from bare and coated surfaces, UV reflectance off surfaces and UV induced breakdown for various insulator material, geometry and angles had to be measured in laboratory settings.

Figure 8 shows a drawing of a test fixture made for HV testing of the full-scale Mini-G prototype insulator. The concave-shaped Rexolite insulator shown here was later fielded in the Mini-G 1 test. Fig. 8 also shows a photo of the hardware setup and a close up of the anode flange after testing.
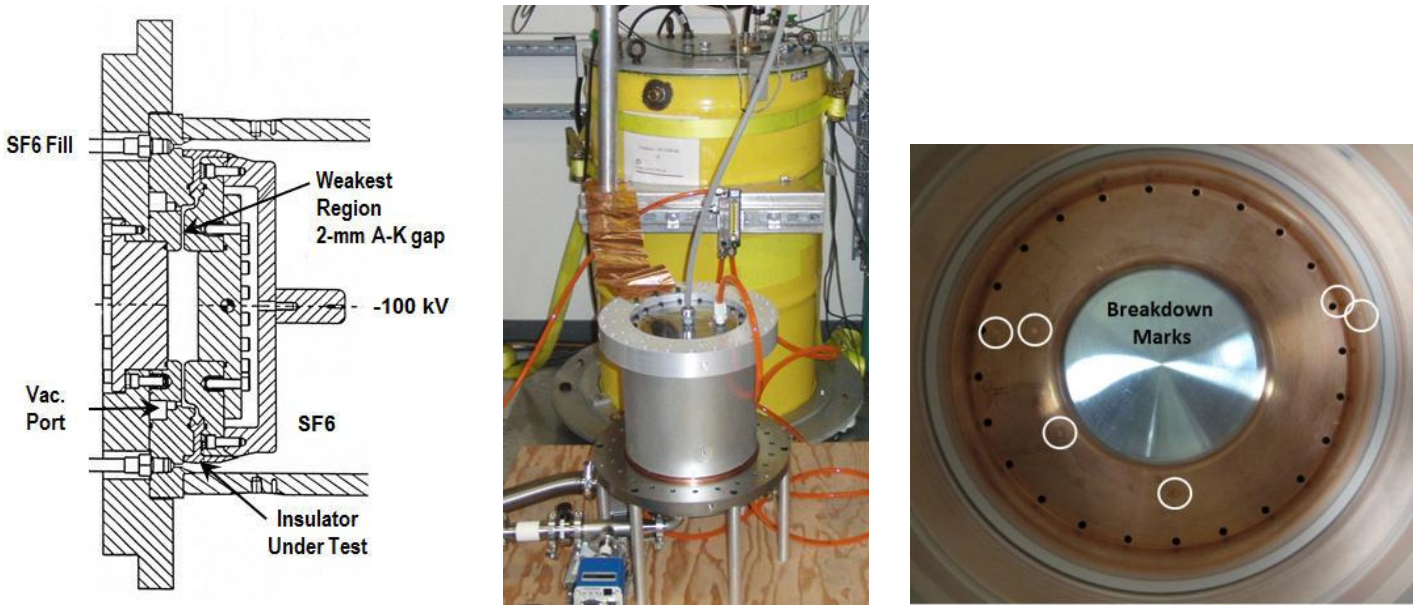

Figure 8. Full size Mini-G vacuum insulator and power flow channel tested. 

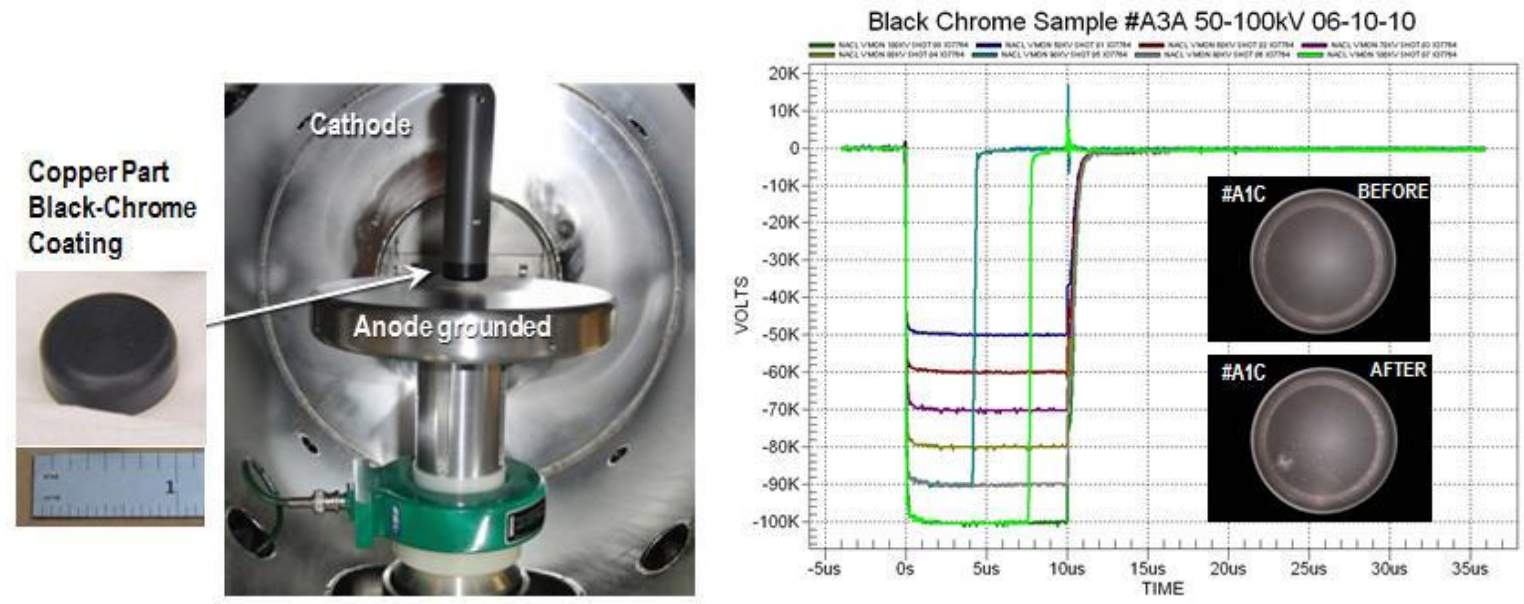

Figure 9. (left) Coatings were tested in a HV vacuum test stand to assess their ability to suppress electron emission. Many samples withstood 100 $\mathrm{kV}$ across the $0.5-\mathrm{cm}$ gap for $10 \mu$ s without breakdown. The example shown at right broke down with only $90 \mathrm{kV}$ applied.

In this testing, the vacuum gap broke down with an applied pulsed voltage of only $20 \mathrm{kV}$. This is to be expected for a $2-\mathrm{mm}$ A-K gap $(100 \mathrm{kV} / \mathrm{cm})$ between bare copper surfaces. After repeating the test six times and disassembling for inspection we were able to identify the six breakdown locations and confirm there was no flashover along the insulator surface. The breakdown in the gap region is not a great concern since we rely on of magnetic insulation in preventing electrons from crossing the channel gap.

A high voltage vacuum test stand was used to measure electron emission and electrical breakdown of different coated surfaces. The test stand incorporates a capacitor discharge unit that can operate up to $100 \mathrm{kV}$ using spark gap switches to adjust pulse width from 1 to 10 us or longer [12]. One of the uses of the test stand was to evaluate coatings to be applied on the cathode surface to suppress electron emission. Figure 9 shows the setup and results from a representative sample of black chrome, which passed a screening test of $280 \mathrm{kV} / \mathrm{cm}$ for $10 \mathrm{us}$.

We also obtained reflectivity measurements on candidate coating materials. A commercial vendor was employed to measure UV light reflection, and determine the bidirectional reflectance distribution function (BDRF) for different surfaces in vacuum over the wavelength range of $200 \mathrm{~nm}$ to $400 \mathrm{~nm}$ (UV). Figure 10 (left) shows measured UV reflectance data from anodized aluminum surfaces prepared by different providers. We have used these coatings in many previous experimental endeavors, primarily for suppression of electron emission from cathode surfaces. Figure 10 (right) shows measured UV reflectance data for black chrome coating on copper. We initially intended to use this coating on the anode surface, but after the HV testing described above, we decided it would be appropriate to use it on the cathode surface as well. The measured reflectance data was used in the FRED modeling as described earlier.

Insulator material and geometry were studied in another test bed which was comprised of an excimer laser $(\mathrm{KrF}$, $248 \mathrm{~nm}, \sim 16 \mathrm{MW} / \mathrm{cm} 2,30 \mathrm{~ns}$ FWHM,), a vacuum chamber (low 1.0E-6 torr), and a negative polarity dc high voltage power supply (up to $-60 \mathrm{kV}$ ) was assembled for insulator $(1.0 \mathrm{~cm}$ thick) testing. See Figure 11a. An in-house designed and calibrated fast capacitive probe (D-dots, $>12 \mathrm{GHz}$ bandwidth) was embedded in the anode electrode underneath
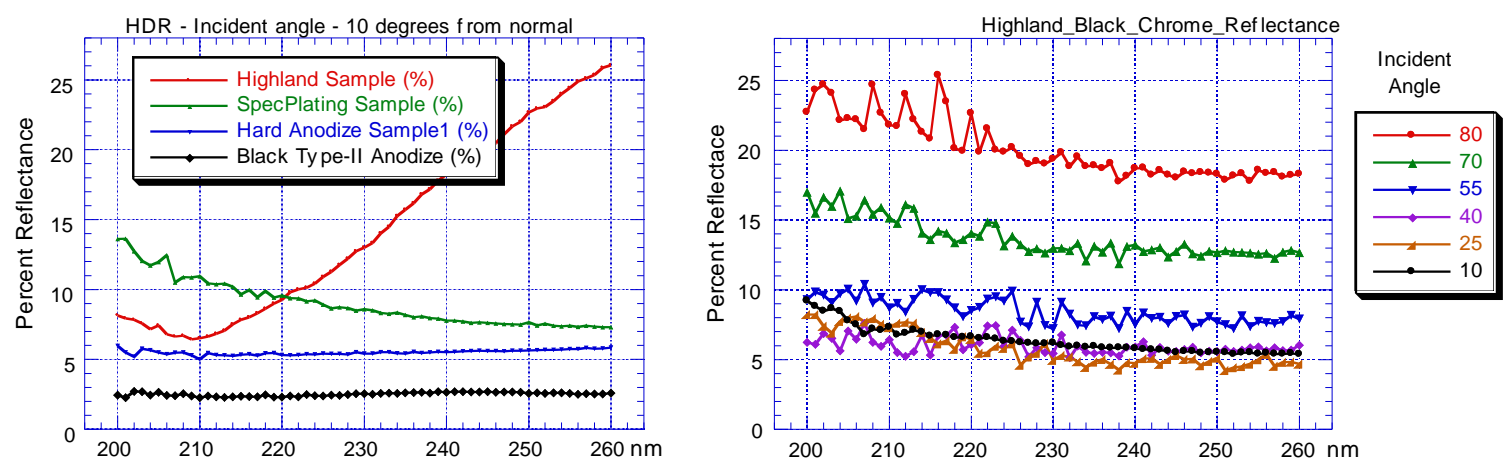

Figure 10. (left) Wavelength dependence on reflectivity for anodized aluminum, (right) Angular dependence on reflectance for black-chrome. 

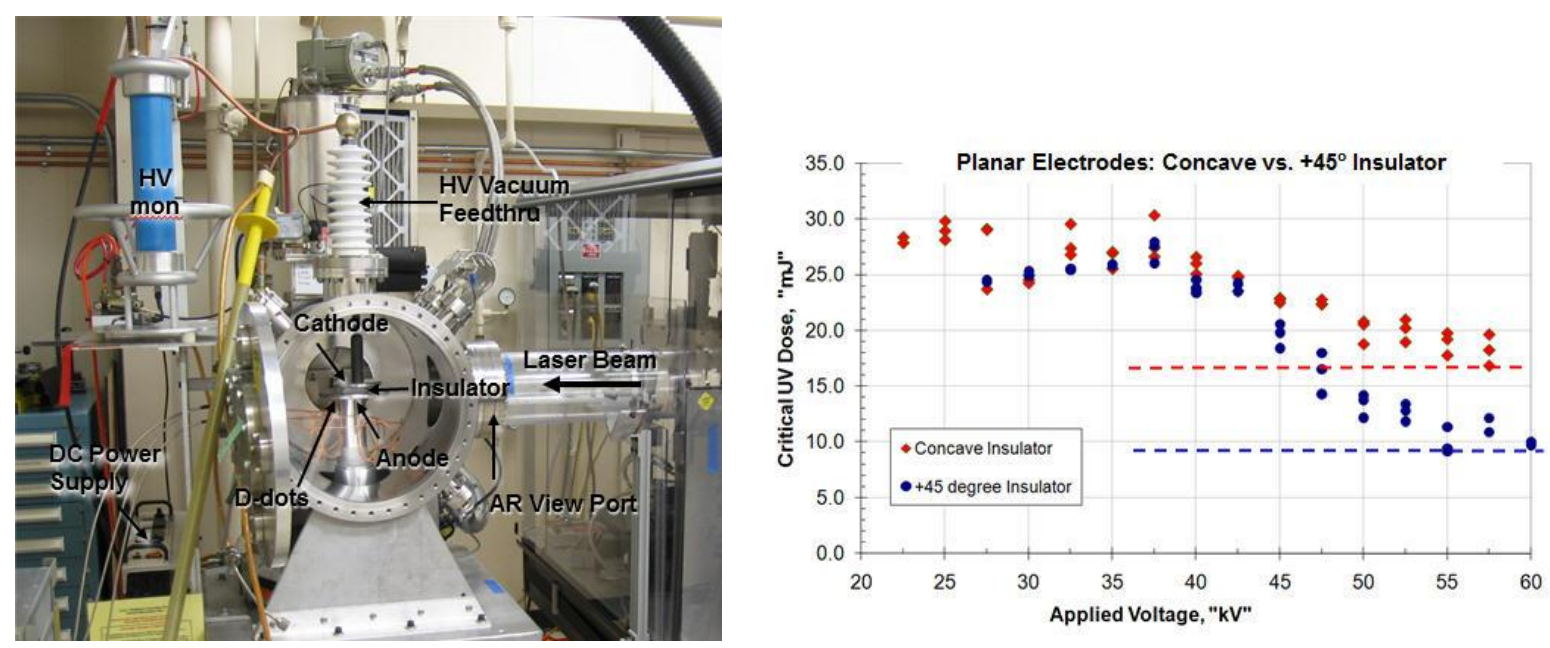

Figure 11. (left) Photo of the test stand for UV induced insulator flashover experiments. (right) Plot of critical UV dose (causing flashover) over a range of applied voltages for Rexolite insulators having concave verses +45 -degree angled sides.

the insulator to determine the time of flashover with respect to time of UV arrival [10] and [13]. The location of the probe was below the UV illuminated section of the insulator. The probe gave highly resolved temporal data and charge data between the UV arrival time and surface flashover time. A beam splitter and commercial energy meter were used to measure the UV fluence for each pulse.

The test bed was utilized for testing of several candidate insulator materials, e.g. High Density Polyethylene (HDPE), Rexolite, Macor and Mycalex, at different cone angles and concave geometries, see Figure 11. The +45 degree Rexolite insulator required more UV fluence to flash; minimum critical fluence of $\sim 13+/-4 \mathrm{~mJ} / \mathrm{cm} 2$, while holding up to $60 \mathrm{kV}$ of DC charge. Figure 11(right) shows that concave insulator did significantly better at higher applied electric field than the +45 degree angled insulator; minimum critical fluence of $\sim 19+/-3 \mathrm{~mJ} / \mathrm{cm} 2$. Based on this result, the concave insulator, of Rexolite, was chosen in fielding of MiniG 1-3. As mentioned, despite this evidence of better improved hold off, partial shorting was experienced in the shots that used concave insulator.

\section{CONCLUSION}

We have developed a high performance vacuum power flow channel for our Mini-G class of generators. Computational codes and laboratory test stands were used in tandem to come up with the most optimized design within the confined physical space and physics requirements. A concave shaped insulator was used to satisfy the ultra-low inductance requirements of Mini-G 1,2 and 3. A conventional 45-degree angled insulator was used in MiniG 4 and subsequent tests. The higher inductance of the latter geometry was accepted in order to reduce the average electric field stress across the insulator and allow the insulator to tolerate greater incident UV. In addition, some changes to the load design on Mini-G 4 were also made to reduce the amount of UV emission. These modifications in effect eliminated the problem of partial shorting experienced in the earlier shots.

The techniques that we employed relied heavily on computational modeling and laboratory testing. These tools were used in the shaping of electrodes and insulators to manage electric field enhancements, coatings on cathode surfaces for low electron emission, baffles in the channel to block UV, and coatings on electrode surfaces to absorb UV. Black chrome plating of the channels walls was chosen over hard anodizing due to simplified manufacturing and lower cost. In addition, it was demonstrated that the high current (10s of MA) of Mini-G in the channel ensured that magnetic insulation stifles the emission of electrons from any charged surface, be it the cathode or the insulator, effectively - preventing the onset of partial shorts in the channel.

\section{ACKNOWLEDGMENT}

The authors would like to acknowledge the outstanding support of associate electrical engineer Ron Speer and associate mechanical engineer Tony Ferriera along with their technical staff who performed experiments in the Pulsed Power Lab and supported field tests. This work performed under the auspices of the U.S. Department of Energy by Lawrence Livermore National Laboratory under Contract DE-AC52-07NA27344. 


\section{REFERENCES}

[1] D. B. Reisman, et al., "Explosive Flux Compression Generators at LLNL," 2012 IEEE International Conference on Megagauss Magnetic Fields (2012).

[2] D. A. Goerz, et al, "Development of High Performance Vacuum Power Flow Interface for Explosive Magnetic Flux Compression Generator Experiments", 2010 IEEE International Conference on Magagauss Magnetic Fields (2010).

[3] [3] J. Hegelberg, "CALE Implementation Guide, UCRL-ID-155540, 2003

[4] R. M. Darlington, T. L. McAbee, and G. Rodrigue, A study of ALE simulations of Rayleigh-Taylor instability, Comp. Phys. Comm., 135 (2001), pp. 58-73. 3

[5] FlexPDE: http://www.pdesolutions.com/

[6] Ansys Maxwell: http://www.ansys.com/Resource+Library/Brochures/ANSYS+Maxwell

[7] Kiuttu G.F., "Calculation of Inductive Electric Fields in Pulsed Coaxial Devices Using Electric Vector Potentials," PPPS-2001, Volume: 2

[8] J. P. VanDevender, D. H. McDaniel, E. L. Neau, R. E. Mattis, and K D. Bergeron, "Magnetic Inhibition of Insulator Flashover," J. Appl. Phys. 53 (6), pp. $4441-4447$ (1982).

[9] Trak: http://www.fieldp.com/

[10] J. B. Javedani, T. L. Houck, D. H. Lahowe, G. E. Vogtlin, and D. A. Goerz, "Insulator Surface Flashover Due to UV Illumination," Proceedings of the 17th IEEE International Pulsed Power Conference, Washington DC, pp. 832-837 (2009).

[11] FRED: http://www.photonengr.com/

[12] M Wilson, D. A. Goerz, and R. D. Speer, "An Elegant Impulser Developed for Flat Beam Injection," Proceedings of the 23rd International Power Modulator Symposium, Rancho Mirage, CA, pp. 88-91 (1998).

[13] J. B. Javedani, T. L. Houck, B. T. Kelly, D. A. Lahowe, M. D. Shirk, and D. A. Goerz, "Ultra-Violet Induced Insulator Flashover," Proceedings of the 2008 IEEE International Power Modulator Conference, Las Vegas, NV, pp. 33-36. 